\title{
Versatile CVD/ALD system
}

This system is instrumental for innovative engineering SRF surfaces and to allow for new breakthroughs:

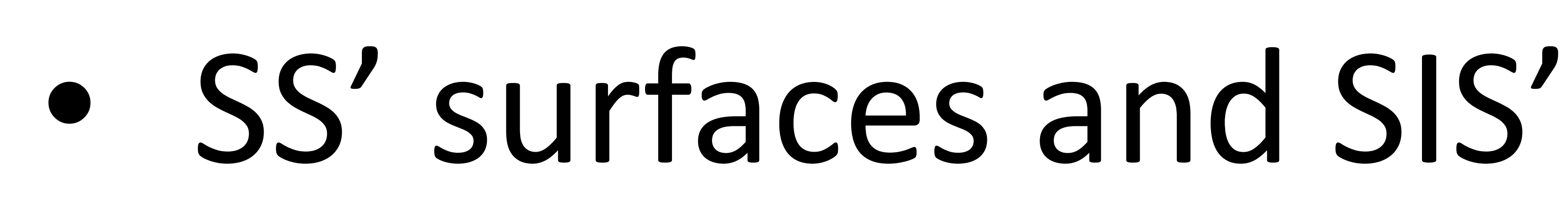
surfaces

- Increasing the accelerating gradient reach

- New A15 superconductors

- Increasing the accelerating gradient and the operational temperature

- New dopant elements

- Increasing the Q-factor and understanding of niobium doping

- Insulators for quantum applications

- Increasing the photon life time in SRF cavities
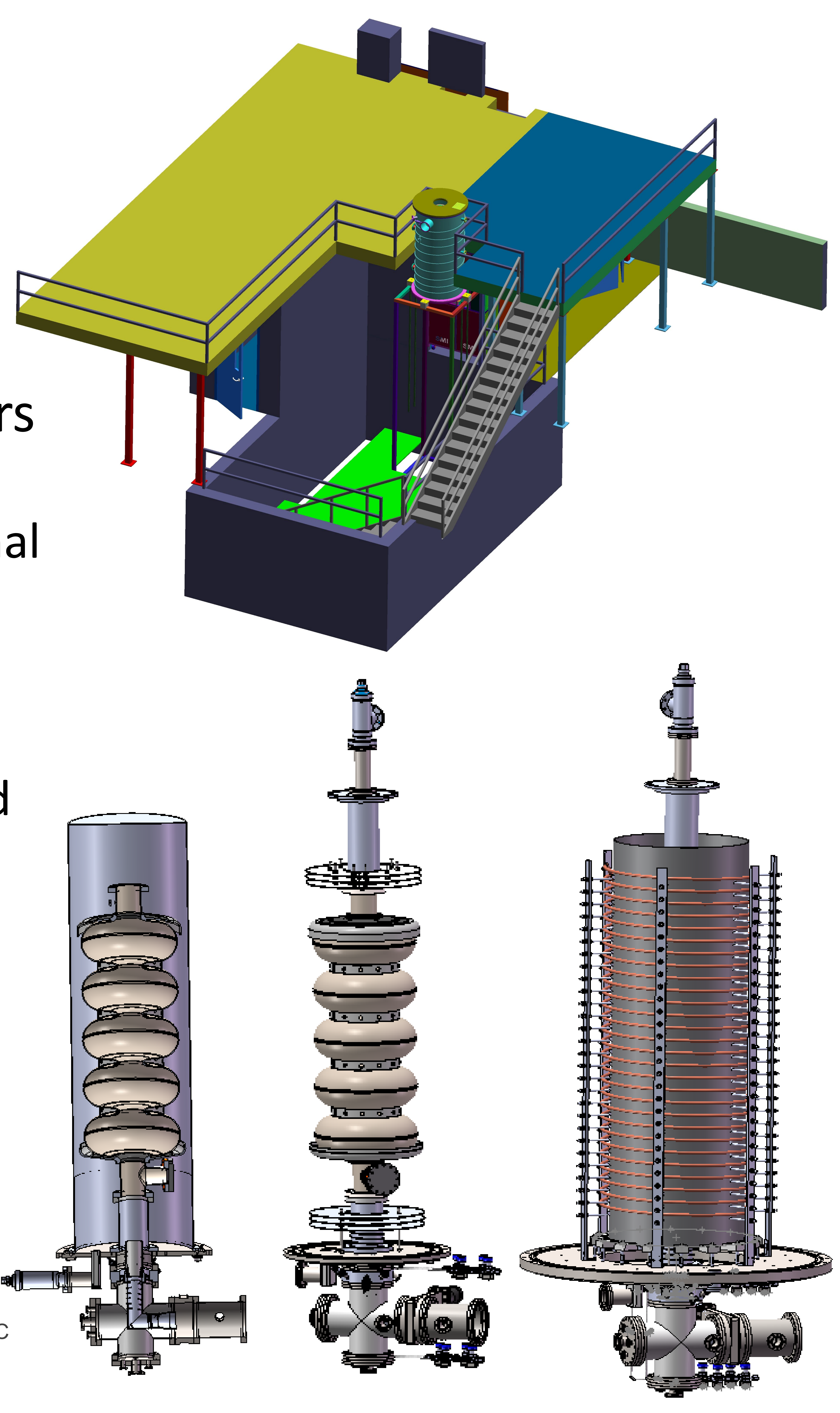